\title{
Hot super-Earths and giant planet cores from different migration histories (Corrigendum)
}

\author{
${\text { Christophe } \text { Cossou }^{1,2} \text {, Sean N. Raymond }}^{1,2}$, Franck Hersant ${ }^{1,2}$, and Arnaud Pierens ${ }^{1,2}$
}

1 Univ. Bordeaux, Laboratoire d'Astrophysique de Bordeaux, UMR 5804, 33270 Floirac, France e-mail: christophe. cossou@u-bordeaux 1.fr

2 CNRS, Laboratoire d'Astrophysique de Bordeaux, UMR 5804, 33270 Floirac, France

e-mail: rayray.sean@gmail.com

A\&A 569, A56 (2014), DOI: 10.1051/0004-6361/201424157

Key words. planets and satellites: formation - protoplanetary disks - planet-disk interactions - methods: numerical - errata, addenda

An error occurred during the production process. An incorrect Fig. 14 was published. The correct Fig. 14 is published below.
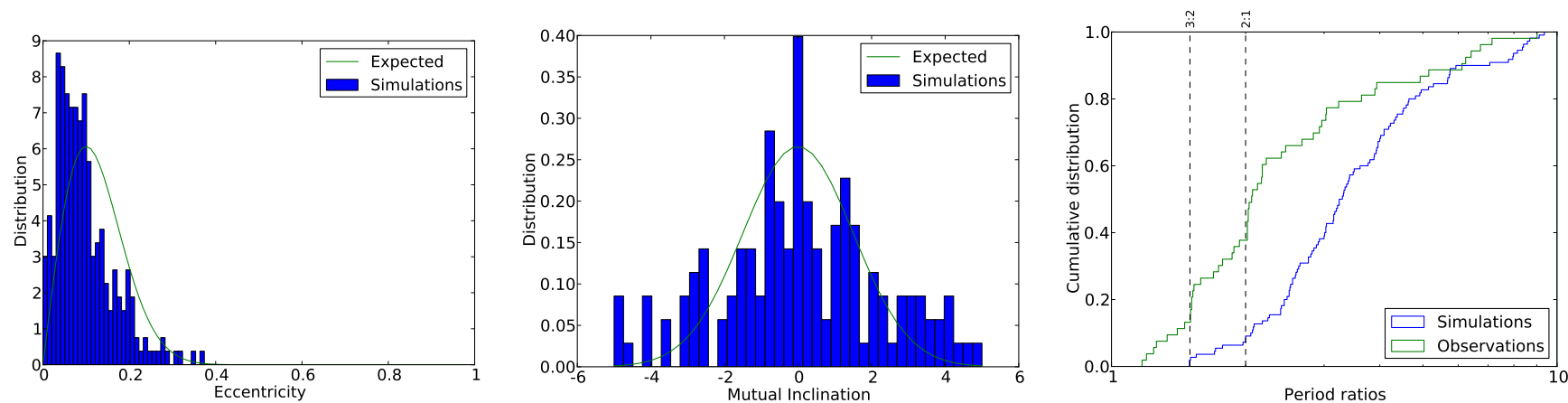

Fig. 14. Comparison between the planets that formed in simulations with dissipating disks and observed extra-solar planets. Both samples are limited to the range $R>1.5 R_{\oplus}\left(3.3 M_{\oplus}\right.$ assuming Earth density) and $p<200$ days. 